\section{Análisis de la problemática socioambiental de Ubaque (Cundinamarca), desde la gestión institucional y la participación}

\section{Analysis of socio-environmental problem in the Municipality of Ubaque (State of Cundinamarca), from the institutional management, and participation}

\author{
Gilberto Vallejo Moreno*, Lizeth Paola Ferro Suárez**, María Camila Awazacko \\ Martínez**
}

\begin{abstract}
*MSc. Gestión Ambiental, Investigador grupo tecnoambiental; Facultad de Ingeniería, Universidad Libre, Bogotá- Colombia (Carrera 70 No. 53-40)

**Estudiante de Ingeniería Ambiental, Facultad de Ingeniería, Universidad Libre, Bogotá-Colombia.

****Estudiante de Ingeniería Ambiental, Facultad de Ingeniería, Universidad Libre, Bogotá-Colombia.
\end{abstract}

\section{Resumen}

El conocimiento y el análisis de la problemática socioambiental de Ubaque, es decir, en el vínculo de la base natural con los procesos sociales y la gobernabilidad, uno de ellos la participación, condujeron a que se validara una hipótesis propuesta: "Los problemas socio ambientales del Municipio de Ubaque en Cundinamarca, y por tanto de la microcuenca del río El Palmar, tienen raíces fuertes en las debilidades de la gestión interinstitucional y en las carencias de la participación ciudadana", mientras que, con el cumplimiento del objetivo "Analizar la problemática socio ambiental del municipio de Ubaque y proponer, a manera de recomendaciones, posibles cursos de acción para mejorar la gestión en pro de su sostenibilidad socio ambiental", las autoridades locales cuentan con una red general de causalidad y una base sólida de información de los problemas más críticos, originadas desde un proceso que incluyó, además del desarrollo de la hipótesis, la revisión de fuentes de información, retroalimentadas con participación de la comunidad, productores y funcionarios, a través de encuestas y entrevistas. La situación no es muy diferente a la de muchos municipios del país de cara, especialmente, a la gestión de las CARS, situándose una debilidad en la gestión de los procesos socioambientales para el ordenamiento ambiental territorial y que compromete, también, a la administración municipal. De alto impacto sería: adoptar un Plan de Manejo Ambiental para la microcuenca, fortalecer la democratización de la información y la comunicación, y articular los Objetivos de Desarrollo Sostenible de la ONU, entre otras recomendaciones propuestas.

Palabras clave: Educación ambiental, gestión socioambiental, problema ambiental, sostenibilidad ambiental, territorio.

\begin{abstract}
Knowledge and analysis of socio-environmental of Ubaque, that is, in the connection of the natural base with social processes and governability, including participation, led to validate a proposed hypothesis: "Socio-environmental problems of the Municipality of Ubaque, in the Department of Cundinamarca, and therefore, El Palmar river micro watershed. Such problems result from interinstitutional management weakness, and lack of citizen participation", while, by achieving the objective of "analyzing the socio-environmental problems of Ubaque, and recommend possible
\end{abstract}

Recibido: 20/01/2016

Revisado: 26/02/2016

Aceptado: 12/11/2016

Correspondencia de autor: Gilberto.vallejom@unilibrebog. edu.co,

lizethp.ferrosunilibrebog.edu.co, mariac.awazackom@unilibrebog. edu.co

(C) 2016 Universidad La Gran Colombia. Este es un artículo de acceso abierto, distribuido bajo los términos de la licencia Creative Commons Attribution License, que permite el uso ilimitado, distribución y reproducción en cualquier medio, siempre que el autor original y la fuente se acrediten.

\section{Cómo citar:}

Vallejo, G., Ferro, L.P., Awazacko, M.C. Análisis de la problemática socioambiental de ubaque cundinamarca, desde la gestión institucional y la aprticipación. UGCiencia 22, 127-147.

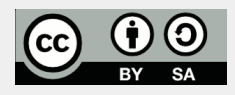


actions to improve management in pro of its socio-environmental sustainability". Local authorities face a general network of causes, and information solid base on the most critical problems, originated from a process which concluded, in addition to hypothesis development, review of information sources, fedback by the community, producers and officials participation, by means of surveys and interviews. Such situation is not different from many municipalities of the country, especially in front of CARs' management. There is a weakness in management of socio-environmental processes, for territorial environmental arrangement, and which also includes the municipal administration. It would be of a great impact to adopt an Environmental Management Plan for such micro watershed, strengthen information and communication democratization, and articulate the UN Sustainable Development Objectives, among other recommendations.

Key words: Environmental education, socio-environmetal management, environmental problem, environmental sustainability, territory.

\section{Introducción}

Conocer la problemática socio ambiental de los municipios colombianos alrededor de la gobernabilidad es identificar los factores que más pueden incidir en su sostenibilidad, al igual que proponer posibles acciones, mientras que, en un municipio como Ubaque, es conocer, también, la situación de gobernabilidad del recurso hídrico de su principal afluente, el río El Palmar, y la sostenibilidad ambiental de su microcuenca. Municipio y microcuenca, surgen como dos espacios, equiparables entre sí1, que a la luz de las normas y los instrumentos de gestión ambiental territorial, v. gr. Esquema de Ordenamiento Territorial (EOT) y Plan de Manejo Ambiental (PMA), permiten, desde una visión integral, planificar y gestionar la sostenibilidad del territorio, no solo ambiental.

En el anterior contexto y en los alcances del proyecto de investigación mayor, mencionado, se centró la investigación, en el siguiente objetivo general: "Analizar la problemática socioambiental del municipio de Ubaque y proponer, a manera de recomendaciones, posibles cursos de acción para mejorar la gestión en pro de su sostenibilidad socio ambiental".

De lo dicho hasta el momento, emergen conceptos que ayudaron a entender la

1. La microcuenca del Río El Palmar ocupa el 93\% de la superficie del municipio de Ubaque, razón por la cual, hablar de la sostenibilidad de dicha unidad hidrográfica natural es referirse a la del municipio. problemática socioambiental del municipio: Por un lado, la gestión ambiental y el problema ambiental, y por el otro, la participación ciudadana y la interinstitucionalidad.

En el seno de las relaciones entre la gestión ambiental y el problema ambiental, se encuentran las acciones u omisiones de los actores institucionales, sociales y económicos que le dan forma al vínculo entre lo social y lo ambiental, y es que en esa relación, es posible abordar la diferencia entre conflicto ambiental y socio ambiental, siendo el último como lo cita Madrigal: "La manifestación de problemas sociales y económicos debido a intereses contrapuestos entre el mundo natural y social" (CAR Cundinamarca-Universidad del Rosario, 2014:7). En el conjunto de problemas identificados, la investigación encontró algunos que bien pueden encajar en la anterior tipificación que a pesar no haber sido desarrollados en este documento, si hicieron parte de la discusión: incremento del libre acceso en el que se encuentran los bienes y servicios ambientales, deficiente identificación de la problemática local y regional y debilidades en el relacionamiento del desarrollo social y económico de la comunidad con los recursos naturales.

Con relación a la participación ciudadana, en la investigación se hizo énfasis en su relación con la educación ambiental, por constituirse, la 
última, en el medio quizá más idóneo, para que los ciudadanos y en general los involucrados, adquieran mayores y mejores niveles de cualificación en pro de la sostenibilidad socio ambiental de sus territorios, además, para actuar con co-rresponsabilidad. Mientras que la interinstitucionalidad, en palabras de Duque Daza, puede ser entendida como "Una alternativa de gestión relacional e interconectada de agentes interrelacionados, frente a modelos auto-centrados e individuales" (CAR Cundinamarca-Universidad del Rosario, 2014,27) siendo los agentes, en este caso, la Administración Municipal y la Autoridad Ambiental.

Dos antecedentes recientes en la jurisdicción de la Car (Cundinamarca), permiten ampliar tanto la discusión como las posibilidades de actuar, participativamente, frente a la problemática socio ambiental de los territorios locales: "Gestión Ambiental, participación ciudadana y sostenibilidad del territorio" de su jurisdicción, y la mencionada publicación, CAR - Universidad del Rosario (2014) ${ }^{2}$.

La investigación realizada, entre los años 2014 (Junio) y 2015, fue de carácter cualitativo - aplicada - , con enfoque particularmente sociológico (área de énfasis: gestión socio ambiental territorial, en diálogo con las ciencias ambientales), y con las siguientes características: participativa, en la medida que fueron involucrados actores de la sociedad civil de Ubaque y funcionarios de la administración municipal, y descriptiva, así como analítica, por cuanto el conocimiento del objeto (la problemática socio ambiental) y el sujeto (el MunicipiodeUbaque)deinvestigaciónloexigían; comparaciones y relaciones también fueron

2. CAR - Pontificia Universidad Javeriana, Bogotá D.C., 2014. Otra experiencia de la CAR, trabajada con la Universidad del Rosario, es: "Construcción de agendas interinstitucionales ambientales. Una mirada desde los derechos humanos" Bogotá D.C., 2014. realizadas. El proceso de dicho conocimiento, por su parte, y más concretamente el problema que afecta al objeto, se sistematizó a través de preguntas de investigación que condujeron a la siguiente hipótesis de trabajo: Los problemas socio ambientales del municipio de Ubaque y por tanto de la microcuenca del río El Palmar, en Cundinamarca, tienen raíces fuertes en las debilidades de la gestión interinstitucional y en las carencias de la participación ciudadana.

La hipótesis además de ser descriptiva es causal, siendo sus características: i. estar mediada por procesos inductivo (a partir de las opiniones y observaciones) y deductivo (desde las preguntas de investigación y la información de fuentes secundarias, hacia las observaciones y opiniones); ii. el grupo poblacional al cual fueron aplicadas encuestas (exploratorias unas, y de comprobación y profundización otras) y entrevistas, fue de 82 personas entre ciudadanos y productores (en 4 de las 19 veredas del municipio, con mayor problemática según la administración y las fuentes secundarias, y en la cabecera urbana) y funcionarios; las exploratorias (15 de ellas) en junio de 2014 y las últimas en mayo de 2015, y un taller para 12 personas entre especialistas y funcionarios; iii. Una línea de tiempo, entre los años 1993 (expedición de la Ley 99) y 2015 (vigencia del Plan de Desarrollo de Ubaque, e informes del Plan de Acción de Corporinoquía), fue necesario establecer, para la revisión cronológica de la información, siendo los años 1998, 2000, 2008, 2012, 2014 y 2015, claves para el análisis de la gestión municipal, y 2000, 2006, 2011 y 2013 para la autoridad ambiental; y iv. los elementos estructurales fueron: a) unidades de análisis: la Administración Municipal, la Autoridad Ambiental y la comunidad, al igual que dos ámbitos territoriales, local (municipio) y regional (conectividad con la jurisdicción de Corporinoquía); b) variables: la participación 
ciudadana en temas ambientales, la gestión en torno al ordenamiento ambiental territorial y la planificación para el desarrollo del municipio, y la gestión en torno a la información y la comunicación; c) y como elementos lógicos, la problemática socio ambiental del municipio, sus relaciones y lo que de ellas emerge.

Las preguntas de investigación fueron: i. La problemática socio ambiental del Municipio de Ubaque ¿tiene relación con la baja ejecución e inversión en proyectos ambientales?; ii. La participación ciudadana en temas ambientales del municipio ¿es débil? y ¿tiene en la gestión de Corporinoquía y la de la Administración municipal, un factor determinante?, y iii. El hecho de que el municipio se encuentre lejano y desconectado geográficamente del centro de las decisiones de Corporinoquía, ¿constituye un factor que también incide en la sostenibilidad socio ambiental del municipio?

\section{Materiales y métodos}

Recordando que la investigación hace parte de un proyecto más amplio del grupo Tecnoambiental, el cual viene siendo orientado por el método Investigación - Acción

En la (...) que se propone una aplicación rigurosa del método científico por parte de un equipo científico técnico, que, a partir de un diagnóstico de la realidad comunitaria diseña la investigación, sus objetivos y el método de la misma, incluyendo la participación parcial de la comunidad, ya sea para la recolección y/o contrastación de los datos de investigación, o para la implementación de las estrategias a seguir" (Miranda D. \&., 2002:12).

En esta investigación se buscó, con la participación de actores, aportar información y análisis de la problemática del municipio al igual que recomendaciones para que sean implementadas a futuro. En este punto, es de resaltar, que a través del convenio UL-Alcaldía, ha venido elaborándose otros proyectos con participación de la comunidad, uno de ellos, el "Diagnostico físico e identificación y formulación de estrategias de manejo para los riesgos ambientales significativos en la microcuenca del Rio el Palmar en el municipio de Ubaque (Cundinamarca)"), y que sirvió, igualmente, para identificar y consolidar la red general de problemas ambientales y generar recomendaciones.

En lo que respecta al proceso investigativo, tuvo cinco momentos: i. revisión, centrada en fuentes documentales originales en su mayoría (textos especializados, documentos y normatividad que recogían la gestión institucional) sistematizadas a través de una base de datos, y páginas web del municipio, la corporación y de otras CAR; ii. situación diagnóstica, con la identificación de la problemática y con ella de los actores, a partir de las variables de estudio y los elementos lógicos; iii. verificación y obtención de resultados: permitieron realizar constataciones y comprobaciones, a través de las fuentes secundarias consultadas y el encuentro de nuevos hallazgos e información, con la aplicación de encuestas, entrevistas, un Derecho de petición, un taller con actores de identificación de problemas críticos (figura 1) y recomendaciones - orientado por la herramienta Matriz de Vester, y observaciones directas; mientras que los resultados, se apoyaron en procesamiento de datos - con salidas gráficas, utilizando herramientas de Excel y de mapas conceptuales - y la descripción de los comportamientos de las variables y los elementos lógicos asociados a la problemática , e igualmente, identificación de hallazgos en fuentes secundarias $\mathrm{y}$, su análisis parcial, por

3. Pinto Sastoque, William Reinel; Pinzón Sánchez, Brayan David (2014). Proyecto de Grado para optar al título de Ingeniero Ambiental de la Universidad Libre. 
comparación con los datos antes referidos y sus comportamientos; iv. análisis y simultáneamente conclusiones y recomendaciones, centrados en la discusión de los resultados de aquellas variables que sustentaban las preguntas de investigación; y finalmente, conclusiones generales.

\section{Figura 1. Red de causalidad de problemas críticos}

\begin{tabular}{l}
$\begin{array}{l}\text { 5. Pérdida de ecosistemas } \\
\text { estratégicos para la regulación } \\
\text { y control de caudales (páramos, } \\
\text { rondas hidricas). }\end{array}$ \\
$\begin{array}{l}\text { 7. Incremento del libre acceso } \\
\text { en el que se encuentran } \\
\text { los bienes y servicios ambientales. }\end{array}$ \\
$\begin{array}{l}\text { 10. Deficiente identificación } \\
\text { de la problemática local y regional. }\end{array}$ \\
$\begin{array}{l}\text { 11. Deficiente identificación } \\
\text { de los riesgos en la zona rural. }\end{array}$ \\
$\begin{array}{l}\text { 17. Red inadecuada de alcantarillado } \\
\text { urbano. }\end{array}$ \\
\hline
\end{tabular}
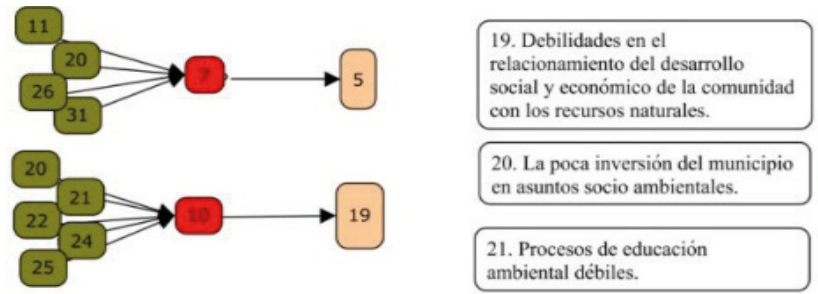

21. Procesos de educación ambiental débiles.

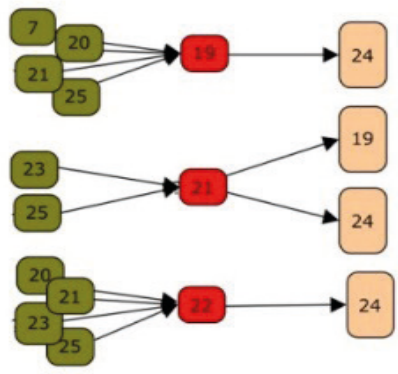

22. Bajos niveles de participación.

23. Bajos niveles de cualificación para ejercer la participación.

24. Debilidad institucional del municipio.

25. Debilidad del SIA de la corporación.

Fuente: los autores

La población, entre tanto, que determinó el tamaño de la muestra para efectos de la aplicación de las encuestas, fue estimada según los siguientes criterios (A. Morillas., s.f.: 20): Muestreo por áreas.

$n=\frac{N z_{1-\alpha / 2}^{2} p q}{(N-1) \varepsilon^{2}+z_{1-\alpha / 2}^{2} p q} \quad$ cálculo de la muestra.

$\mathrm{n}=$ muestra

$\mathrm{N}$ : tamaño de la población

Z: valor de la tabla de probabilidad según la campana de Gauss para un $95 \%$ de confiabilidad es 1.96
P: Proporción esperada es del 50\%

E: Error de precisión (5\%)

q: es $(1-0.05=0.95)$ se le resta el porcentaje de error.

La población para el municipio de Ubaque para el año 2015 fue de 6.116 habitantes (proyecciones DANE).

$$
\begin{gathered}
n=\frac{(6116) *(1.96)^{2} *(0.5) *(0.95)}{(6116-1) *(0.05)^{2}+(1.96)^{2} *(0.5) *(0.95)} \\
=72.15 \text { Encuestas }
\end{gathered}
$$

Las encuestas, por su parte, tuvieron preguntas tanto cerradas (dicotómicas y de respuesta múltiple) como abiertas, mientras 
que las entrevistas fueron no estructuradas, recordando que en total fueron realizadas 82 (15 exploratorias en una primera jornada).

\section{Resultados}

\section{Resultados de las variables o los problemas claves identificados}

\section{Bajos niveles de participación}

Laparticipación y conella la educación ambiental y la gestión que por ellas se haga, constituyen factores de primer orden para calificar el impacto de la gestión institucional, un indicador de gobernabilidad, mientras que, en el seno de sus relaciones, está la co-rresponsabilidad con la que la participación se ejerce. En Ubaque, la situación de la Educación Ambiental (EA) pasa por la baja inversión y debilidades de la gestión, y con relación a la red de problemas

"El diagnóstico (...) identificó que el principal problema de la cuenca del Río Negro, es la gran debilidad en educación sobre el tema ambiental y la valoración de los recursos naturales (...), situaciones comunes en las áreas rurales de la mayoría de microcuencas" (POMCA RIO NEGRO, 2006, pág. 136)

Siendo una responsabilidad, la EA no era considerada específicamente por la administración, al menos en el Plan de Desarrollo Municipal 2012 - 2015 y tampoco en el Plan de Accion Programado 2015. Se asociaba a la productividad, al ambiente y otros sectores, coincidiendo con lo expresado por la comunidad a la pregunta: ¿Qué programas tiene la Alcaldía frente a las problemáticas ambientales que aquejan al municipio?, no reconociéndola como un programa, sí como actividades de capacitación, como lo muestra la figura 1 . Un $32 \%$ de peso tuvo la capacitación, y el $34 \%$ dijo no saber o no reconocían su existencia.(figura 2 ).
El banco de proyectos del Informe de Gestión 2014 para 2015, mostró en cambio la intención de mejorarla, en su vínculo con la participación ${ }^{4}$ (ALCALDIA DE UBAQUE, pág. 76).

\section{Figura 2}

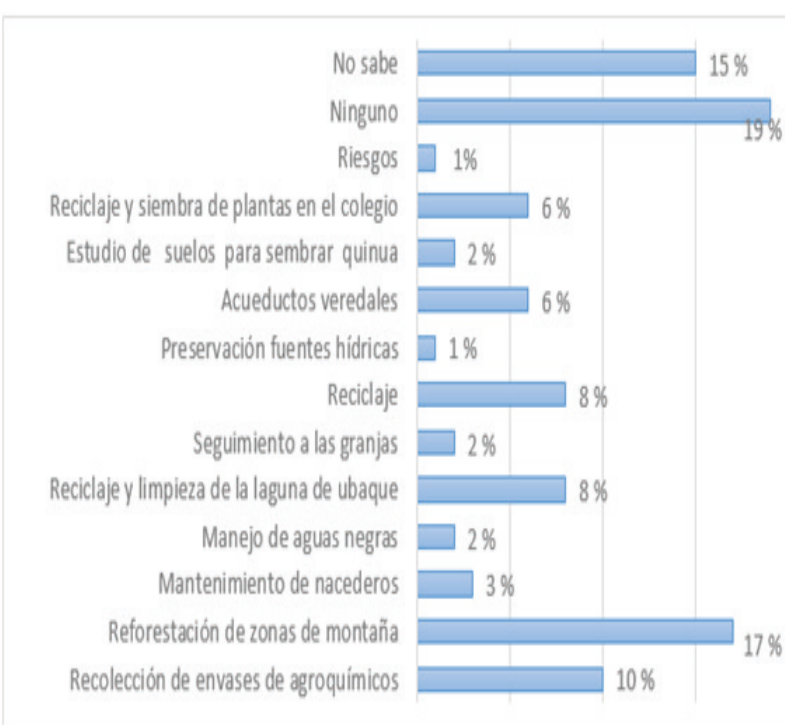

Fuente: los autores.

Por su parte Corporinoquía, a quién por norma le obliga un mayor compromiso, lo reportado

no permitió conocer, por ejemplo, las cifras de la inversión ${ }^{5}$; la información cualitativa, en cambio, sí aportó algunas referencias (CORPORINOQUIA, 2012, pág. 185):

Al analizar la "educación y comunicación ambiental para el fortalecimiento institucional y la participación comunitaria", en dicho Plan, se dedujo que es débil, por la "Baja cobertura en las estrategias y deficiencias en la de educación ambiental preventiva" (CORPORINOQUIA,

4. De los 40 proyectos, en su mayoría correspondieron a infraestructura vial, solo uno a medio ambiente, tres a aspectos sanitarios y ninguno a territorio, mientras que en participación (integración ciudadana), cinco.

5. los Gastos de Inversión de la entidad en los 5 grandes Programas, por ejemplo, no permitieron conocer como fueron asignados en cada región ni municipio, a pesar que proyectó el "Fortalecimiento de la educación ambiental para la sostenibilidad" (Proyecto 040101, pág. 136), con el apoyo de PRAE y una red de PROCEDA. 
2012, pág. 13) a pesar que ha tenido una importante evolución, como es el surgimiento de los Proyectos Ambientales Universitarios (PRAU) y el fortalecimiento de la educación para las organizaciones sociales.

Al comparar los planes de 2013 y 2015, faltó más visibilidad al impacto de los procesos y los recursos invertidos, p. e., frente a la adaptación al cambio climático, tema desarticulado de los Proyectos Ambientales Escolares (PRAE) y los Proyectos Comunitarios de Educación Ambiental (PROCEDA) y más aún de los PRAU y del EOT. En el marco del Plan de Gestión Ambiental Regional (PGAR), es notable el énfasis de los PRAE sobre los otros; además se encontraron inconsistencias en las cifras, cuando se comparan con otras de educación, p. e., de Ubaque. Y Al analizar la estructura de los Planes Operativos de Educación del (Corporinoquía, 2013) se confunden las unidades con los indicadores de las metas y de las actividades, y en el seguimiento, no se hace énfasis en el impacto.

Ahora, cuando se mira la problemática y en general la sostenibilidad socio ambiental (SSA) del municipio, en relación con la situación de la participación, esta es determinante. Uno de varios indicios, es la influencia en los demás problemas críticos, como se puede leer en la red general de problemas. A propósito, el Plan de Desarrollo Municipal (PDM) 2012 - 2015 señala que en el municipio: "No hay una sola organización ambiental" (Alcaldia de Ubaque, 2012:143).

En la difusión del proceso del EOT, se encontró otra evidencia (figura 3). A la pregunta, ¿ha participado en la elaboración del nuevo EOT del municipio?, solo el 2\% respondieron afirmativamente, coincidiendo en que, en marzo de 2014, asistieron a una actividad de divulgación - no de socialización - de la Alcaldía (invitación por la página web).

\section{Figura 3}

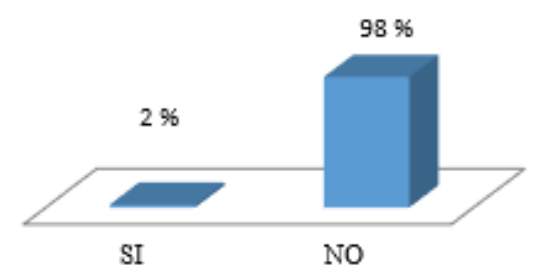

Fuente: los autores.

Otros referentes son: la baja presencia de colectivos sociales, en el taller (febrero 27 de 2014) de "Priorización de Microcuencas municipio de Ubaque"6 (una lista de asistencia corrobora lo observado), y la opinión de la Corporación recogida en el (Nullvalue, 2004)., al calificarla como "deficiente, motivada hacia sus intereses", es decir, con baja co-rresponsabilidad.

Con relación al actuar de la comunidad frente a las problemáticas ambientales (PA) (figura 4), el $45,7 \%$ dijo que de forma diligente, los funcionarios lo pusieron en duda (47,8\% coincidieron en que no) aunque aceptaban que un bajo porcentaje lo hacía $(15,2 \%)$, y otros (el 37\%), motivados por algún interés. El reconocimiento de la comunidad a la gestión de la Alcaldía como fue la existencia de al menos 10 programas, jugó, sin embargo, a favor de la gestión.

Sobre el proceso de Ordenamiento Ambiental Territorial (OAT) para la planificación del municipio, y el acceso a la información y la

6 Programado por la Empresa de Acueducto y Alcantarillado de Bogotá (EAAB) dentro del programa "Corredor de Conservación Lobo Guerrero - Chingaza - Sumapaz". 
comunicación, se encontraron otras evidencias:

a) En el marco de la gestión de Corporinoquía, está el Plan de Ordenamiento y Manejo (POMCA) del Río Negro?

Figura 4. Actuar de la comunidad frente a las problemáticas ambientales del municipio.

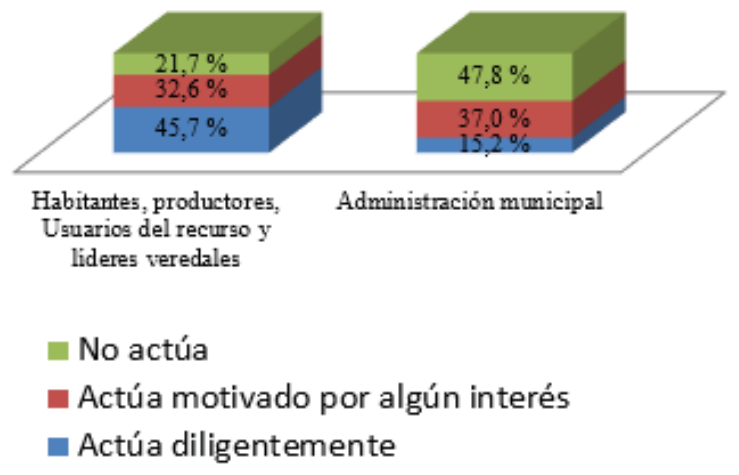

Fuente: los autores.

Por un lado, la baja representación de la comunidad en los talleres de aprestamiento y con relación al Consejo de Cuenca, el documento de Aprestamiento, poco o nada fundamenta sobre él, más allá de mencionar la existencia de un Anexo. $\mathrm{Y}$ en la Formulación (CORPORINOQUIA, SF, pág. 136) solo el $8(42 \%)$ de los proyectos abordaron la participación; mientras que la Prospectiva (POMCA, 2006, pág. 18), aunque ahondó en los escenarios, no cumplió del todo con los requisitos del Decreto 1729 de 2002.

b) Otro escenario, fue el de los indicadores y las metas fijadas por el Modelo Programático del PGAR. Presentó inconsistencias para una mejor gestión de la participación, frente a los requisitos de las Resoluciones 0643 y 0964 (Ministerio de Ambiente Vivienda y Desarrollo Territorial MAVDT, 2004) y (MAVD, 2007)

7. La participación fue dimensionada, en su momento (año 2006 de elaboración del estudio), por la Guía Técnico Científica para el POMCH (POMCA) c) Con relación a la gestión de la información y la comunicación: La situación del internet en el municipio, por ejemplo, fue expresada y corroborada por el PDM 2012 - 2015 (Alcaldia de Ubaque, pág. 208) al citar: El Municipio en la actualidad no brinda a la comunidad un servicio público de las redes de internet, (...) a esto se suma que los establecimientos educativos cuentan con computadores pero no con el servicio, (...).

Una revisión (Marzo 25 de 2015) a su página web (instrumento de las Tecnologías de la Informaciòn y la Comunicación - TIC), dijo que la problemática tenía que ver, también, con la robustez y la pertinencia, pues adolecía de información para la mayoría de las opciones de consulta; en los indicadores, no se encontró medio ambiente y la actualización era de marzo 16 de 2009, y no registró comentarios de la ciudadanía.

Las deficiencias encontradas fueron respaldadas por la encuesta. A la pregunta: la "Alcaldía da a conocer los proyectos relacionados con el medio ambiente (...)", el 58,7\% dijo estar enterados, y al restante $41,3 \%$ no les llegaba la información o no les interesaba el tema, como se evidenció en las observaciones directas. Contrasta que el $100 \%$ de los funcionarios dijeran que sí los daban a conocer ${ }^{8}$ (figura 5). De otro lado, el $55.2 \%$ de los habitantes y el $17,4 \%$ de los funcionarios, indicaron que los medios de mayor difusion de la información correspondían a otros diferentes a los modernos, el perifoneo (de la iglesia) y el voz a voz (siendo frecuente el problema de distorsión de la información); el $15,5 \%$ y el $52,2 \%$, respectivamente, que se hacía con panfletos (tipo folletos), y el 22,4 \% y

8. Entre el porcentaje que dijo estar enterado, se encontraron los docentes de las escuelas o colegios y algunos líderes de las Juntas de Accion Comunal (JAC) de las veredas o de los distritos de riego, por el conocimiento directo de los proyectos implementados por la administración; en otros casos, por el voz a voz o circulares de la Alcaldía. 
el 13,0 \%, destacaron la emisora; los habitantes dijeron no usar la pagina web del municipio, $\mathrm{y}$ el 6,9\% indicó, que la alcaldia no usaba ningun medio de difusión de la información y que las reuniones con líderes veredales eran los más ágiles, junto con los anteriores (figura 6).

\section{Figura 5}

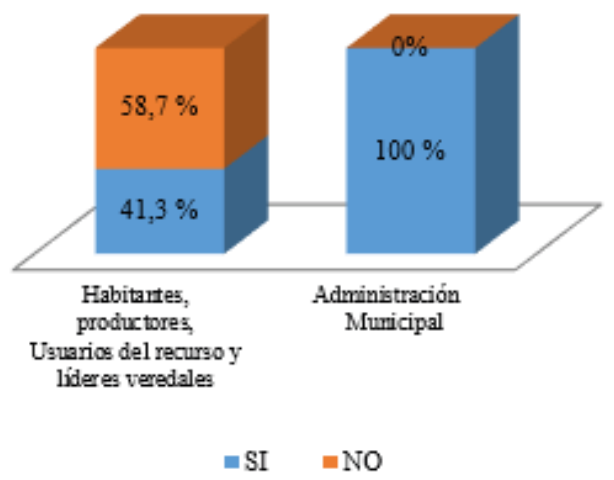

Fuente: los autores.

La situación hasta ahora expuesta, se presentaba, sin quitarle merito a que se tenían proyectados: el fortalecimiento de la infraestructura ciudadana (Alcaldia de Ubaque, pág. 216), gobierno en línea y uso interno y externo de medios electrónicos (Alcaldia de Ubaque, 2015, pág. 77 a 79), y por parte de Corporinoquía, la Ventanilla Integral de Trámites Ambientales en Línea (VITAL), entre otras acciones, cuestionada por la administración municipal (Prado, 2015).

Figura 6 ¿Cuál es el medio de mayor difusión que utiliza la alcaldía para brindar información a los habitantes del municipio sobre proyectos relacionados con medio ambiente?

Finalmente, se revisaron, el PGAR, el Plan de Acción 2012 - 2015 y la página Web de la corporación, recordando que, metodológicamente, se aplicaron criterios de consulta y calificación.
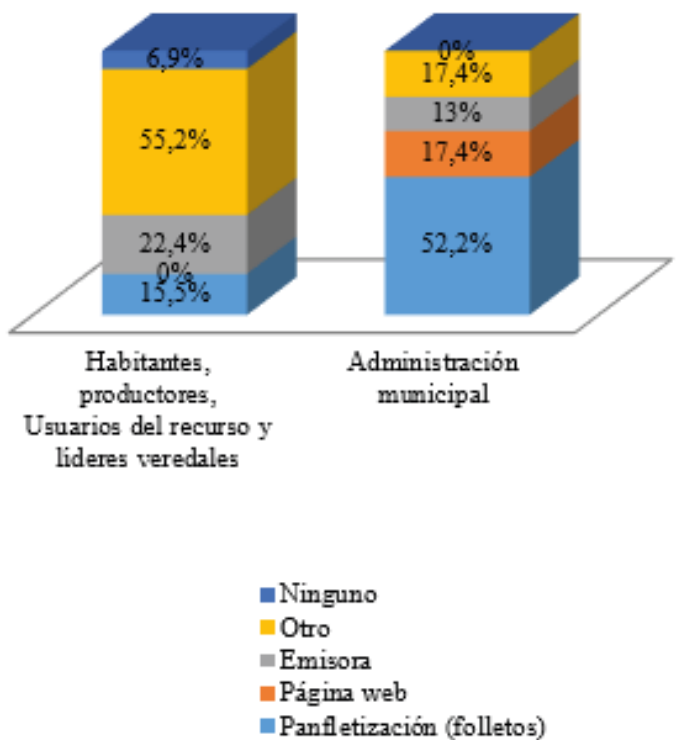

Fuente: los autores.

Sobre la cantidad y distribución de las subsedes, los datos reportados evidenciaron que la Autoridad Ambiental (AA) cumplía con lo dispuesto por la Ley 99 de 1993 (Art. 33), al tener sede en cada región, pero, al comparar la cantidad de oficinas, las cifras se vieron en desventaja con, p. e., la CAR Cundinamarca, y Corpoguavio con los mejores índices, razón que motivó a que desde la Alcaldía de Ubaque, se halla pensado en adherir a ella.

$\checkmark \quad$ De su página web, se evidenciaron deficiencias en la migración de datos, p. e., en temas de contaminación. De acuerdo con el Derecho de Petición (2014): “A Nivel regional (...) y local, municipio de Ubaque, no se había articulado interinstitucionalmente una red de información ambiental que hiciera efectiva la información de apoyo a los procesos de gestión ambiental regional, pero la Corporación (...) creó la ventanilla VITAL, usada especialmente para denunciar conflictos ambientales" "a los cuales Corporinoquía responde meses después", (Prado, 2015). Y de la Unidad Ambiental de Cáqueza, se resalta que limitaba la inscripción de nuevos usuarios. 
El resultado de las encuestas arrojó que algunas causas tenían que ver con el bajo acompañamiento de Corporinoquía, p. e., en capacitación a los productores y en EA. A la pregunta: ¿Con cuánta frecuencia se ha reunido en los dos (2) últimos años con representantes de la Corporación para tratar asuntos ambientales del municipio? el $17 \%$ de los productores dijo haberlo hecho de 3 a 6 veces, y el 52,2\% en ninguna, y aceptable para los funcionarios (figura 7).

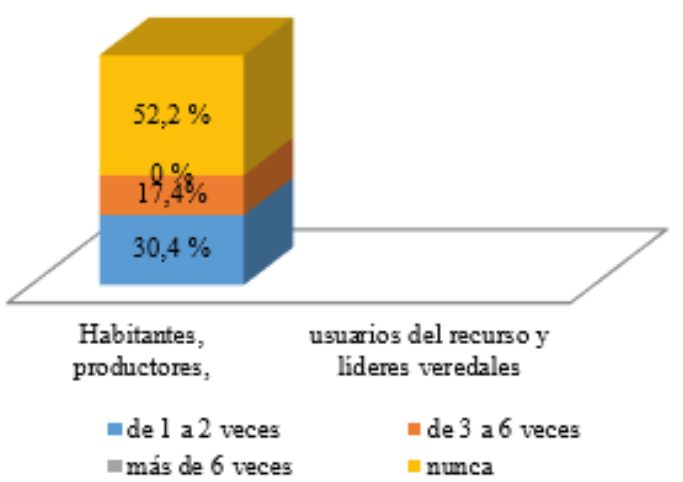

La opinión de la entidad y la información reportada en el Plan de Acción, dieron a conocer, que "por falta de personal, cuatro sedes no son suficientes para hacer el debido acompañamiento a cada uno de los municipios de toda su jurisdicción" (Nullvalue, 2004), lo cual coincidió con las debilidades reportadas, también, en el plan:

No existe un sistema de información de la jurisdicción y hay deficiencia en el sistema de gestión documental; la corporación cuenta con recursos propios limitados (...), lo que hace que la infraestructura locativa sea inadecuada e insuficiente, y deficiente capacidad operativa y logística para atender una jurisdicción tan grande y compleja ambientalmente (CORPORINOQUIA, 2013, pág. 13); y la página web se encuentra muy saturada y sin una organización clara haciendo que para los usuarios promedio el acceso sea difícil, y prácticamente imposible para usuarios con discapacidad (CORPORINOQUIA, 2013, pág. 122)

Debilidad de relaciones institucionales municipio - autoridad ambiental, y el bajo reconocimiento, en el municipio, de la gestión de la autoridad ambiental.

Las relaciones Municipio - Corporinoquía, con la gestión y el control ambiental y de los recursos naturales, no han pasado por su mejor momento en los últimos años afectando, también, a otros municipios de su jurisdicción en Cundinamarca.

Las fracturas de dichas relaciones, como se ha observado, constituyen una de las principales causas de otros problemas críticos (o claves), llegando a incidir fuertemente en la calificación, la cual también ha afectado a la Autoridad Municipal (AM).

a) De la gestión del municipio, los resultados de las encuestas dijeron: Algunas causas tenían que ver con la poca inversión para mejorar ambientalmente las actividades productivas, calificada como regular (39\%) como se ha evidenciado, y un alto porcentaje (61\%) no respalda la gestión en este sentido, resultado contrastado por la opinión positiva de los funcionarios, con el 87\% (figura 8). Una revisión al Informe de Gestión 2014 (Alcaldia de Ubaque, 2015, pág. 76), y que proyectó la inversión para el 2015, como se recordará mostraba una tendencia a la baja en inversión en temas ambientales, lo cual le dio la razón a los pobladores. 
Figura 8. ¿La alcaldía realiza inversiones en el municipio para mejorar ambientalmente las actividades productivas?

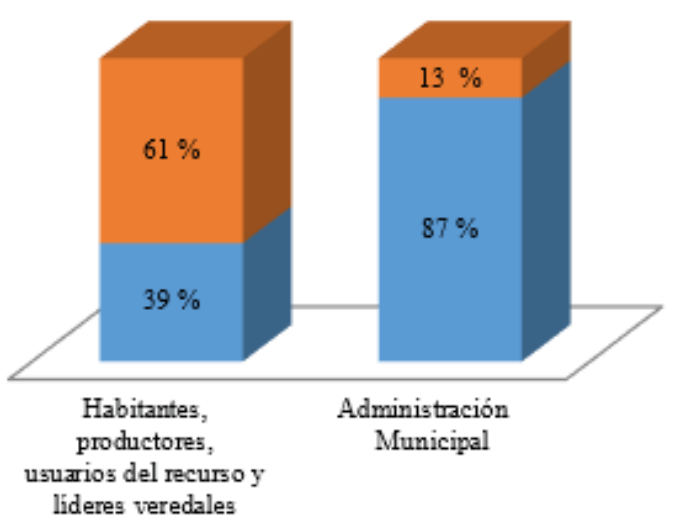

$\because \mathrm{SI} \quad \mathrm{NO}$

Fuente: los autores

Con relación al compromiso de los funcionarios, fueron formuladas dos preguntas:

¿Considera que el nivel de compromiso de los funcionarios de la administración municipal es consecuente con el grado de las problemáticas ambientales del municipio?

Como se deduce de la figura 9, las opiniones se encontraron divididas entre lo que pensaba la sociedad civil y los funcionarios: mientras el $43,4 \%$ de los primeros consideraron consecuente el nivel de compromiso de los funcionarios, el $46,7 \%$ de aquellos lo calificaron con alto grado, presentándose una situación similar en la valoración más baja del compromiso; la coincidencia se dio en la calificación de grado medio, $54,4 \%$ y $53,3 \%$, respectivamente.

Figura 9. Considera que el nivel de compromiso de los funcionarios de la administración municipal ¿es consecuente con el grado de las problemáticas ambientales del municipio?

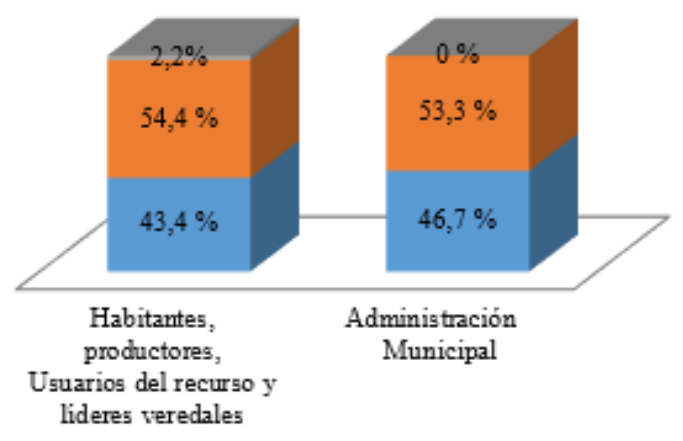

"Incons ecuente
$=$ Medianamente cons ecuente
$=$ Consecuente

Fuente: los autores

Mientras que, conocer la fuerza de la participación de los funcionarios y su opinión sobre la de la comunidad, en asuntos de interés para el municipio, aportó otro elemento de análisis. La mayoría (el 50\%) de los encuestados, coincidieron en que era poco fuerte, y fuerte para el $21 \%{ }^{9}$.

Contrastan las cifras, con la marcada debilidad de la calificación dada a la participación de la comunidad (Ver figura 10).

b) Por el lado del proceso de ordenamiento territorial en el municipio y con él su EOT, la situación no es ajena a lo que en Colombia ha sucedido con los Planes de Ordenamiento Territorial (POT) ${ }^{\mathbf{1 0}}$, siendo dos

9. Un hecho permitió inferir, sin embargo, el mejoramiento a futuro de la AM en asuntos ambientales. Se trata del cambio de la Umata a una Secretaria de Desarrollo Rural y Ambiental.

10. No se puede desconocer que el ordenamiento territorial en el país ha contado con más problemas que logros, (...). Se puede considerar que la inexperiencia frente al tema, la escasa información confiable y la falta de recursos en general, no permitieron un proceso de formulación de planes armónico, ordenado y efectivo". 
Figura 10 Participación de la comunidad y los funcionarios en asuntos de interés para el municipio

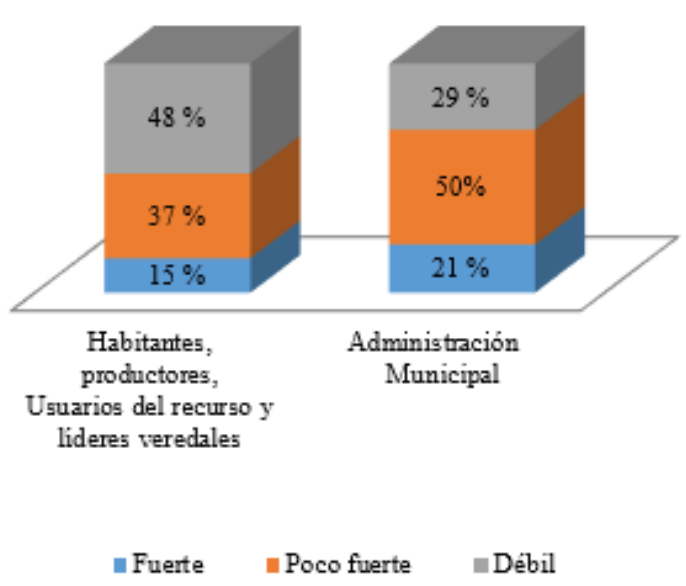

Fuente: los autores

factores la calidad de la información y la articulación del mismo (el EOT) con otros instrumentos. Para Ubaque y los municipios colindantes como Choachí, se evidenció un escenario con visos de conflicto, por la inminente intervención de las obras del proyecto vial Perimetral de Oriente ${ }^{11}$, caso que se discutirá posteriormente.

La visión de la corporación confirma lo analizado (CORPORINOQUIA, 2012, pág. 75 y 76)

c) De la relación administración - corporación, el Plan de Desarrollo 2012 - 2015 de entonces no dejó bien parada la gestión de aquella en el municipio al calificarla como regular (Alcaldia de Ubaque, 2012, págs. 163, tabla 48). El desconocimiento de la AA cuando se le preguntó sobre: ¿cuáles programas tiene la

11. Un viaducto sobre el río El Palmar de $95 \mathrm{~m}$. de longitud, la instalación de una estación de peaje con bascula de pesaje, la posible utilización de dos fuentes de materiales, la localización de dos zonas para la disposición de materiales sobrantes ZODME, y la intervención de 6 veredas, por el mejoramiento y sección transversal y rehabilitación de los accesos sur y norte al casco urbano del municipio y de la vía actual que comunica con Choachí. alcaldía frente a las problemáticas ambientales que aquejan al municipio? es otro indicio de las debilidades en la comunicación y la baja sinergia interinstitucional (Nullvalue, 2004).

d) Un asunto aún más concreto, también con visos de conflicto, que ofreció indicios sobre el estado de dichas relaciones, fue el inconformismo, no solo de la administración de Ubaque, también las de los municipios aledaños, por las deficiencias en la atención.

De acuerdo con El Tiempo.com ${ }^{12}$, en el año 2004 dos titulares llamaron la atención: "Protestas en contra de Corporinoquia" y "Cundinamarca se rebela"

No queremos hacer parte de Corporinoquia esa es la frase que constantemente repiten los mandatarios, quienes advierten que esa entidad ambiental no sólo tiene su sede en un sitio lejano, en Yopal (Casanare), sino que además se quejan de que no se les tiene en cuenta sino para conseguir votos para la elección de sus directivas y para el pago del 1\% del impuesto predial. En cuanto a ejecución de proyectos ambientales, el apoyo es nulo, dijeron los mandatarios.

El secretario de gobierno de Ubaque y la directora de la Umata ${ }^{13}$ ratificaron las quejas de los 9 alcaldes de la región, mientras que en defensa de los señalamientos, el director de la entidad:

Sostuvo que en la actualidad Corporinoquia, mediante (...), realiza una inversión inicial de 1.200 millones de pesos para (...) proyectos de reforestación de las cuencas de los ríos Negro y Palmar. El disgusto de los alcaldes obedece a la no elección del candidato que ellos apoyaban para el cargo de revisor fiscal (Nullvalue, 2004: 1).

12. http://www.eltiempo.com/archivo/documento/MA M-1591904 (agosto $10 \mathrm{y}$ 24 de 2014)

13. En conversación telefónica de junio 19 de 2013, además apoyaba el "cambio a otra autoridad llegando a mencionarse Corpoguavio". 
La anterior situación fue identificada, también, por la AA (POMCA RIO NEGRO, 2006, pág. 136) y reconocida por la misma entidad, en su Plan de Acción y en la respuesta al Derecho de Petición que se le formuló en octubre de 2014.

Otra de las soluciones que ha surgido, por parte de la entidad, fue la instalación de una oficina en Chipaque:

(...) trasladada a Cáqueza (...), sin embargo, esto tampoco les ha dado resultado. Rosa D. Agudelo, alcaldesa de Guayabetal, dijo que esas oficinas son solamente intermediarias, pero no han servido porque la gestión hay que hacerla directamente en Yopal. La plata que nosotros le giramos a Corporinoquia, que es el 1.1 por ciento del impuesto predial, la deben utilizar en conservación de cuencas y apoyo a reforestación, pero la verdad hace falta más presencia de ellos en Cundinamarca, señaló (Tiempo, 2004).

Otro titular del mismo periódico, que recogió resultados del libro "La Región de la Orinoquia", dijo que "la Corporación no se inscribe dentro de un propósito regional y regionalizador" como consecuencia de "La relación (...) con los municipios y departamentos, calificada como artificial, porque no responde (...) para resolver problemas".

e) Del proceso de formulación del POMCA y del Plan de Acción 2012 - 2015 de la entidad, surgieron otros factores para revisar su gestión: "El proceso de ordenación de la cuenca (...), ha presentado múltiples tropiezos y limitantes que han retardado su desarrollo" (CORPORINOQUIA, SF, pág. 21), y del Plan de Acción, se destacó que el licenciamiento ambiental estuvo ausente, lo que a juicio de la Contraloría General de la República - CGR (Tiempo, 2015) involucra tanto a la Autoridad
Nacional de Licencias Ambientales (ANLA) como a las CARs del país ${ }^{14}$.

f) $\mathrm{Al}$ conjunto de situaciones tratadas, se le suman dificultades en lo político y el orden público, dos amenazas como la AA lo reconoce "Injerencia politiquera dentro del desarrollo corporativo y la presencia de organizaciones al margen de la ley" (CORPORINOQUIA, 2012, pág. 13), a pesar de que la entidad “(...) ha generado información útil para la región" 15 y el municipio (Nullvalue, 2004).

Para finalizar, se retomarán el Plan de Acción, en lo que tiene que ver con la inversión y el alcance de algunos proyectos, y la página web y el sistema de información (SIAR - QUIA para efectos de la investigación), desde la forma y el alcance de los indicadores, además, unas noticias de la CGR. Se encontró:

a) No hubo desagregación de la inversión para cada municipio, asunto que "no le corresponde a la entidad" Derecho de Petición sin desconocer la inversión en Educación y Participación, aunque baja. (CORPORINOQUIA, 2012, pág. 26)

b) Una revisión a 4 de los Planes Operativos Anuales de Inversión (POI) de los años 2013 a 2015 de Corporinoquía, dijo que no había indicios de elaboración o proyección de PMA para microcuencas y/o acuíferos, como lo establece el Decreto 1640 de 2012.

14 "No hay control eficaz a las licencias ambientales y las deficiencias recaen en la ANLA y en las CARs". Al respecto, es necesario puntualizar: que si bien los últimos arreglos legales del país le han quitado protagonismo a las AA regionales con la creación de la Autoridad Nacional de Licencias Ambientales -ANLA-, también es cierto, que al momento de encontrarse la investigación en fase de análisis, se produjo la Sentencia C-035 de 2016 de la Corte Constitucional que declara inexequible el artículo 51 del PND (Ley 1753 de 2015), con lo que le devuelve funciones a las CAR.

15. Como es el POMCA del río Negro, Plan de Manejo del páramo Cruz Verde, Formulación del Plan de Ordenamiento y Reglamentación del Recurso Hídrico del Rio el Palmar, Plan de Ordenamiento Forestal, Cartografía Temática y básica del municipio; y se establecieron los Objetivos de Calidad para los cuerpos de Agua Receptores de Vertimientos de Aguas Residuales en la Cuenca del Rio Negro, (...)". 
c) Con relación a la gestión en torno a la información y la comunicación:

1. El Decreto 1200 de Abril del 2004, fija el "Diseño del Sistema de Información Ambiental para Colombia-SIAC" (Art. 9). CORPORINOQUIA "ha hecho un avance a nivel del PGAR, sin embargo, todavía tiene retos en la generación de información ambiental para su jurisdicción, y le hace falta articularse como Sistema de Información Ambiental Territorial (SIAT) (Nullvalue, 2004).

2. La Resolución 0643 de 2004 , establece los indicadores mínimos del artículo 11 del Decreto 1200 de 2004. Al respecto, el PGAR dice (CORPORINOQUIA, 2013, pág. 191): "En cumplimiento de lo establecido en el Decreto 1200 de 2004, se tiene previsto incorporar al sistema, los indicadores mínimos de gestión estipulados en las Resoluciones No. 643 de 2004 y 964 de 2007 y de igual forma, los indicadores definidos por las entidades del sector ambiental (MADS, institutos de investigación del SINA) así como la Contraloría General de la República".

3. El sistema de indicadores del PGAR es un bosquejo, y en su fundamentación, adoleció de información que los soportara frente a las "Hojas Metodológicas de los indicadores mínimos" de la Resolución O643 de 2004 (Art.8).

d) La CGR, a través de los (Informes del estado de los recursos naturales) de los años 2006-2007, 2010 - 2011 y 2012 a 2015, aportó otros hallazgos de la gestión de la entidad, que confirman, en varios casos, la situación de Ubaque y la región:

4.
5. Deficiencias en el Sistema de Control Interno en diferentes áreas de la entidad.

6. Bajo avance en la ejecución de planes, programas y proyectos.

7. Retraso en el ordenamiento de Cuencas hidrográficas.

8. Inadecuado manejo y gestión presupuestal.

9. Continúa el deterioro de ecosistemas estratégicos y de la biodiversidad en la región.

10. Revisando los períodos 2006 - 2007 y 2010 - 2011 la gestión mejoró, y para el año 2012 se presentaron desfavorables 3 de los 5 hallazgos, también en 2013 y 2014, además de incluir un bajo avance en la ejecución de planes, programas y proyectos. Señaló también la Contraloría:

11. Existe falta de control y vigilancia por parte de CORPORINOQUIA a la gestión y manejo externo de los residuos hospitalarios y similares; fueron encontrados (...) errores o inconsistencias que afectan la razonabilidad de los estados financieros. Tampoco existe rigor en el control y seguimiento por parte de la entidad, a actividades mineras de extracción de materiales para construcción, vertimientos y concesiones de agua.

\section{Discusión de resultados}

Decir que las principales causas de la problemática socioambiental del municipio, tienen raíces fuertes en factores de la gestión de Corporinoquia y la Administración Municipal, fue un descubrimiento hasta cierto punto esperado por la investigación, por lo que en el país ha sucedido con la gestión ambiental de las CARs quienes comparativamente, por norma, tienen el mayor peso de la 
gobernabilidad ambiental, lo que condujo a que la investigación, con un alcance principalmente local, haya enfrentado limitaciones de la información disponible, dicho de otra forma, por la condición regional de la jurisdicción de la AA que marca una generalización de la misma, v. gr., en las escalas de la cartografía que la acompañan. Lo anterior, sin desconocer que a las administraciones municipales les obliga, también por norma, gestionar el ordenamiento territorial (en coordinación con las AA), un proceso hasta ahora débil en Ubaque y con un importante peso sobre la problemática socioambiental (PSA).

Es en el marco de esa problemática y los factores asociados, es que a continuación se hace un análisis centrado en las preguntas de investigación, incluyendo algunas recomendaciones a que dieron lugar la información disponible y los resultados, siendo los temas centrales, por lo tanto: la problemática socio ambiental, la participación ciudadana en asuntos ambientales, la inversión en proyectos ambientales por parte de la Administración Municipal y la Autoridad Ambiental y por último, la gestión de las mismas en pro de la Gestión Ambiental (GA).

\section{La problemática socioambiental del municipio}

Los PSA que aquejan al municipio son diversos, como amplias son sus potencialidades $y$, sensibles, varios ecosistemas que la sustentan, lo que llevó a descubrir, que abordar el conocimiento y la conservación de los recursos naturales $(\mathrm{RN})$ naturales de su territorio, es un trabajo por demás complejo. Del conjunto de problemas claves (figura 1), la participación y con ella la EA, constituyen el centro de gravedad de los demás, y los relacionados con las debilidades de la gestión, los principales motores que los dinamizan, dicho de otra manera, el resultado confirma una de las preguntas de investigación, la relación causal de la gestión hacia la participación, dos componentes fundamentales para la gobernabilidad, recordando que la EA, como proceso que también es, debe hacer parte integral y sine qua non de la participación, siendo la apropiación social del conocimiento, su vínculo más directo.

Es así como, en la medida que se dé una participación cualificada y con co-rresponsabilidad, los involucrados con la SSA del territorio, seguramente contribuirán de forma importante, no solo con el conocimiento y la solución de la problemática, también con sus potencialidades.

\section{La inversión en proyectos ambientales por parte de la administración municipal y la autoridad ambiental}

Recordando que la pregunta busca relacionar la PSA con la ejecución e inversión en proyectos ambientales, los resultados, aunque no contundentes, sí permiten inferir una incidencia moderada, con tendencia a ser baja en el caso de la AM.

Primero está la opinión de los encuestados: poca inversión de la Alcaldía y el énfasis en proyectos de intervención sobre los ambientales, con una leve mejoría en educación y participación a través de infraestructura para la integración ciudadana, y el poco acompañamiento de la corporación. Los requerimientos de índole económica que le hiciera la corporación al municipio en el Informe de Seguimiento Agenda Ambiental del año 2011 (págs. 4, 6 y 9) por deudas vencidas, constituye otro hallazgo.

Por parte de la AA, está la falta de desagregación de la inversión para cada municipio, y los hallazgos de la Contraloría. Sobre estos últimos, en su relación con la gobernabilidad 
y la problemática identificada como clave, hay por decir que en cualquier ámbito territorial la conservación o degradación de los ecosistemas y en general los RN y el ambiente, tienen en la eficiencia y la eficacia con que los recursos se manejen, dos asuntos fundamentales, y que bajo esa premisa, la situación de Ubaque no tiene por qué ser diferente, al menos en lo fundamental.

Una puesta en perspectiva de los hallazgos de la inversión de la AA en los Planes de Acción y del impacto que se pudiera esperar de las metas e indicadores de línea base del PGAR, permite decir, en cambio, que si ellos tuvieran como objeto la SSA del municipio, los beneficios serían importantes, por destacar, entre otros: la inclusión de la gestión del riesgo en el OAT y definir una estrategia para la consolidación del patrimonio ambiental municipal, y estudios para la definición de umbrales ecológicos e impacto al recurso hídrico, de proyectos de infraestructura. En el anterior contexto, no es garantía, p. e., que la inclusión de la gestión del riesgo se logre, si contiene el mayor porcentaje de procesos e instrumentos, sin que medien criterios de inclusión.

También se identificaron circunstancias que pudieran clasificarse como de índole político, y que inciden en la discusión de los asuntos económicos y la gestión de la entidad. La construcción de confianza entre la administración municipal y la corporación será fundamental para una buena gestión y el mejoramiento de la opinión ciudadana, y los cambios que se vislumbran en el país, en torno al pos conflicto, seguramente lo serán para la injerencia de grupos armados.

Dos recomendaciones se derivan de todo lo analizado: un esfuerzo de la AA, por mejorar la efectividad de la información de inversiones en cada municipio, empezando por la del POMCA, y mejorar los indicadores fiscales, y que se haga un fuerte acompañamiento al municipio, en la articulación de los componentes socioambientales del EOT, POMCA y PD y, a la gestión del riesgo, se le dedique un capítulo especial.

\section{La participación ciudadana en asuntos ambientales}

Los resultados obtenidos, permiten deducir que dicha participación en Ubaque es débil, y que en las deficiencias de la gestión se encuentra uno de los factores determinante, siendo la inversión y lo que se haga por la EA, dos asuntos importantes. El panorama es aún más complejo, cuando se acude a la co-rresponsabilidad con la que la misma se ejerce; trabajar en ella, mejoraría significativamente el conocimiento y la conciencia de la comunidad sobre el patrimonio ambiental, al igual que la percepción de la gestión realizada por la institucionalidad.

Otros indicios se encuentran en la influencia de la participación hacia los demás PA claves, y sobre la dinámica como se ha venido ejerciendo el OAT del municipio y de la región, léase los procesos del EOT y el POMCA: los bajos niveles de asistencia a eventos y los aspectos cualitativos de la participación, mientras que, en la democratización de la información y de la misma participación, pueden estar las respuestas para mejorar los indicadores de confianza interinstitucional y de opinión.

Las inconsistencias y debilidades mencionadas, parten de las dificultades de los usuarios para la consulta y solicitud de información en las páginas web, y tienen en los indicadores y metas de los informes de planificación, gestión y acción, unos instrumentos para ser mejorados, si se busca medir el impacto de la gestión. 
Una lectura a los resultados de los problemas claves, influenciados por las debilidades de la participación y de la educación, permite analizar:

Que la deficiente identificación de la problemática local y regional, también de las potencialidades, en su relación con la educación, es corroborada por la AA cuando expone, como amenaza, la "deficiencia en conocimiento y valores ambientales para una efectiva participación en la gestión ambiental regional y local" (CORPORINOQUIA, 2012, pág. 13 y 132), "situaciones comunes en las áreas rurales de la mayoría de microcuencas" como también lo son los problemas asociados a la capacitación de los campesinos "que no puedan armonizar los valores ambientales con la realidad económica y social que a diario asumen o en ocasiones con las simples prácticas culturales adversas a la protección y conservación" (CORPORINOQUIA, 2012, pág. 136), una de ellas la del Río El Palmar, considerada "como de muy alta prioridad de acción en el POMCA" (CORPORINOQUIA, 2012, pág. 13). La sinergia entre esos problemas, conlleva a que se presente incremento del libre acceso a los bienes y servicios ambientales, y debilidades en la relación del desarrollo socio económico de la comunidad, con los recursos naturales. Los resultados de la investigación apoyan lo expuesto.

De lo analizado surgen como recomendaciones que Corporinoquia en coordinación con el municipio, cuanto antes elabore, e implemente el PMA para la microcuenca del río El Palmar, y en ese propósito, aprovechar los proyectos formulados por el POMCA en armonía con el EOT (actualizado), fijando una meta, restablecer, consolidar y mantener una organización de base del Consejo de Cuenca - CC; la futura Secretaría de Desarrollo Rural y Ambiente (SDRA) del municipio, podría ser un buen apoyo. Además, consolidar una red de aliados ambientales, conformando una red PRAE - PROCEDA - PRAU, desde el Comité Técnico Interinstitucional de Educación Ambiental (CIDEA), y fortalecer el sistema de metas e indicadores de la participación, siendo la medición del impacto un criterio.

\section{La gestión institucional e interinstitucional local y regional y las condiciones geográficas en las que ellas se dan}

Preguntarse, aquí, por la influencia de la gestión de la administración municipal y de la AA en la PSA de Ubaque es reafirmar que constituye el factor de mayor peso. Y es que las relaciones entre ellos, en el marco de la gestión.

Se debe recordar, claro está, que las dos instituciones han adelantado acciones y han planificado otras en atención a algunos de los problemas, solo que persisten asuntos por mejorar y fracturas que componer en la gobernabilidad, uno de ellos, romper las barreras geográficas y hasta políticas para una mejor gestión " (...) dentro de un propósito regional y regionalizador".

De la situación expuesta emerge una reflexión: si bien es cierto que hay una relativa larga distancia entre Ubaque y Yopal, mucho más cercana con la sede de Cáqueza y, la información y las comunicaciones, en teoría se encargan de mejorarlas y acortarlas, también lo es, que la planificación por la SSA debe ser el compromiso interinstitucional que rompa, a través del recurso hídrico y las unidades hidrográficas, las barreras físicas y las de las comunicaciones.

Sobre la forma, como se ha dado el proceso de ordenamiento territorial (OT), dicho de otra manera, el del EOT actual y su actualización, hay por reflexionar que no son muy distintas a 
la mayoría de los municipios del país, y que no en pocos casos entran en discordancias y hasta en conflictos con otros instrumentos para el OAT y el desarrollo.

¿Y cuál puede ser una consecuencia de tales debilidades y desarticulaciones para Ubaque? Un caso, tiene que ver con los comentarios del directordela Agencia Nacional de Infraestructura ANI, al referirse al proyecto de la Carretera Perimetral de Oriente: "Si la herramienta no está actualizada, es responsabilidad directa del municipio" (EL ESPECTADOR, 2015, pág. 1), en respuesta a la preocupación, en Choachí, por la cercanía $\left(200 \mathrm{~m}\right.$.) de la vía al casco urbano ${ }^{\mathbf{1 6}}$. Es de recordar, que Ubaque también será intervenido por las obras de dicho proyecto.

De otro lado, en el centro de la problemática que afecta la participación y la EA, se encuentra la Democratización de la Información y la Comunicación. Al respecto, se recomienda:

Que la Alcaldía revise a fondo la página web, pues aunque posee 25 Opciones de consulta muy pocas despliegan información, incluyendo el Plan Ambiental, haciéndola más amigable y rigurosa, actualizándola permanentemente, y mejorando cuantitativa y cualitativamente los comentarios y en esa dirección los indicadores de ambiente y territorio. De igual forma, aprovechando los planes de Gobierno en el uso de medios electrónicos, mejorar la cobertura del internet en la zona rural.

Entre tanto, para CORPORINOQUIA, lo deseable es que se incremente el número de funcionarios de la oficina de Cáqueza, se agilicen los tiempos de respuesta de la ventanilla VITAL, y hacer más amigable y eficiente la página web.

En cuanto al sistema de información y su articulación con la gestión, el OAT y la 16. http://www.elespectador.com/noticias/bogota/los-desacuerdos-via-perimetralde-oriente-articulo-607685. Consultado, mayo 30 de 2016. sostenibilidad ambiental territorial, un plan de mejoramiento debe considerar, además de las acciones en favor de la comunicación, al menos:

Subsanar las deficiencias en las metas y los indicadores, especialmente de línea base ambiental, atendiendo a la normatividad, y en ese contexto, que sean propuestos indicadores para drenajes y lagunas (v. gr. Grande de Ubaque), hidrobiológicos unos, y para caudal ecológico y evaluaciones de línea base .

Priorizar para el municipio algunas metas: la implementación de mecanismos de tecnologías de producción limpia, adoptar Esquemas de Pago por Servicios Ambientales, articular la página web del municipio al centro de investigación e información SA de la Orinoquia, hacerlo parte de la capacitación de organizaciones en gestión del riesgo ambiental, y mejorar, a través de indicadores de impacto, el uso y aprovechamiento del agua de cuerpos hídricos loticos y lenticos.

$Y$ en el marco de los instrumentos para el OAT y el desarrollo del municipio, que las dos instituciones adopten y articulen los Objetivos de Desarrollo Sostenible de la Organización de las Naciones Unidas (ONU), entre ellos: agua limpia y saneamiento; producción y consumo responsables, acción por el clima, vida de ecosistemas terrestres y paz, justicia e instituciones sólidas, por el impacto positivo hacia a la SSA de Ubaque y la región, un reto a la vez para la gobernabilidad.

\section{Conclusiones}

Los problemas socioambientales -PSA del Municipio de Ubaque y por tanto de la microcuenca del Río El Palmar, tienen raíces fuertes en las debilidades de la gestión de la administración municipal -AM y más aún de la autoridad ambiental -AA, y como consecuencia, 
en las carencias de la participación ciudadana siendo la educación ambiental -EA un componente fundamental. Lo encontrado condujo a comprobar, por un lado, la hipótesis de trabajo y por otro, a inferir que el resultado hasta cierto punto era el esperado, en el contexto de lo que en el país ha venido sucediendo con la gestión ambiental -GA, especialmente de las CARs, a lo cual también están obligados los entes territoriales.

Factores deíndole político marcan las similitudes y ¿hasta la injerencia de grupos armados?, al igual que la prevalencia de la inversión en proyectos de intervención en infraestructura, por parte de los Alcaldes, muy por encima de la asignada a los proyectos socio ambientales, característica que ayudó a sustentar una de las preguntas de investigación.

Por lo tanto, es necesario que se mejore la GA, en cabeza de la AA, con: la desagregación de la información sobre inversiones en cada municipio y los índices de razonabilidad de los estados financieros, el seguimiento y el control, la escala de la información de los estudios, la fundamentación y alcance de muchas de las metas e indicadores de línea base ambiental y de gestión, particularmente para el recurso hídrico y los recursos naturales que lo sustentan, al igual que las demandas; y con lo anterior, el fortalecimiento del sistema de información ambiental, en coordinación y con el compromiso y esfuerzo, igualmente, de la AM, y según se vayan dando los resultados esperados, muy seguramente seguirán surgiendo los apoyos de los aliados, entre ellos las mismas empresas, organizaciones de productores y la academia.

Los resultados y lo analizado entorno a ellos, específicamente lo relacionado con la participación y la gestión institucional e interinstitucional, condujeron a que se confirmaran las otras preguntas de investigación.
Ubaque comparte tanto potencialidades como limitaciones ecológicas y PSA con los municipios limítrofes, especialmente con Choachí, Fómeque y Cáqueza, sin embargo, históricamente, los destinos de la GA del último (jurisdicción de Corpoguavio), han sido preferidos por los otros, aunque con una esperanza para ellos, que Corporinoquia, en más de una ocasión ha reconocido las debilidades de su gestión y también ha producido algunos instrumentos y estudios, específicos, que con mayor seguimiento y control seguramente mejorarán la situación de su sostenibilidad socio ambiental -SSA-.

El territorio del municipio de Ubaque es complejo, por la diversidad de los PSA y de sus potencialidades y por la sensibilidad de varios de los ecosistemas que las sustentan, como lo es, enfrentarla deficiencia en conocimiento y valores ambientales, para una efectiva participación en la gestión ambiental regional y local, y en esa medida, contrarrestar, el incremento del libre acceso a los bienes y servicios ambientales y las debilidades en la relación del desarrollo socio económico de la comunidad con los recursos naturales. Surgen, así, la gestión o apropiación social del conocimiento, como una estrategia fundamental, al igual que la oportunidad y un reto para la gobernabilidad, cual es que la administración y la corporación, adopten y articulen, al OAT, los Objetivos de Desarrollo Sostenible de la ONU.

Un factor ha sido visto por Corporinoquia como una amenaza para su propia gestión, y que en la investigación se interpretó, como la necesidad de incluir, dentro de la categoría de los Temas de la página web, el licenciamiento ambiental.

El estado en que se encuentra el EOT del municipio y la existencia del POMCA del Río Negro, se presentan como dos oportunidades 
para la construcción de una hoja de ruta, en pro de la SSA del municipio, en armonía con la región, $\mathrm{y}$ en beneficio de la gestión interinstitucional.

Una dificultad y una exigencia mayor tuvo que enfrentar la investigación. Por un lado, las debilidades pre-existentes de la participación en el municipio para afrontar la opinión de los actores, y por otro, las limitaciones de la información para el nivel local, pues mucha de la información disponible estuvo marcada por la generalización.

\section{Referencias bibliográficas}

A. Morillas. (s.f.). Muestreo en poblaciones finitas, apuntes de clase. Recuperado de http://webpersonal.uma.es/ morillas/ muestreo.pdf: https:/www.google.com. co/?gfe_rd=cr\&ei=lpkov46rgjazmagihl syca\#q=antonio + morillas + muestreo + en + poblaciones + finitas

Alcaldia de Ubaque. (abril de 2012). Plan de desarrollo municipal 2012 - 2015 (tabla 48). Recuperado de http://www. ubaque-cundinamarca.gov.co/apc-aa$0303461323365643963383734623963 /$ plan-de-desarrollo-2012-2015-modificado.pdf

Alcaldia de Ubaque. (2015). Informe de gestion vigencia 2014. Recuperado el 30 de abril de 2015

\section{Car Cundinamarca-Universidad del Rosario.}

(2014).Elementosparalaconstrucciónde agendas interinstitucionales ambientales en casos de conflictos ambientales. En 1. C. Valencia, construcción de agendas interinstitucionales ambientales. Una mirada desde los derechos humanos (pág. 27). Bogotá D.C. Universidad del Rosario.
Car

Cundinamarca-Universidad

del

Rosario. (2014). Revisión conceptual de los confilctos socio-ambientales para la construcción de nuevas tipologías. Construcción de agendas interinstitucionales ambientales. Una mirada desde los derechos humanos" Bogotá D.C. Universidad del Rosario.

Congreso. (22 de diciembre de 1993) Diario oficial 41146 de diciembre 22 de 1993: http://www.alcaldiabogota.gov.co/ sisjur/normas/norma1.jsp?i=297

Corporinoquia. (24 de noviembre de 2006). Pomca rio negro. Corporinoquia, casanare. Yopal: Corporinoquia.

Corporinoquia. (2006). Revision y ajuste linea estrategica formulacion de programas y proyectos pomch rio blanco - negro - guayuriba. Corporinoquia. (2011). Seguimiento agenda ambiental. Corporinoquia, Casanare, Yopal.

Corporinoquia. (2012). Plan de accion 2012 - 2015. Casanare. Yopal: corporinoquia. Por una region viva: http://1. corporinoquia.gov.co/images/archivos/ $\mathrm{pdf} / \mathrm{plan} \% 20 \mathrm{de} \% 20$ a c c i on $\% 20$ $2012 \% 202015 \% 20 . p d f$

Corporinoquia. (2013). Plan de gestión ambiental regional 2013 - 2025. Corporinoquia, Casanare. Yopal: Corporinoquia.

Corporinoquia. (2013). Planes operativos anuales de inversion 2013 a 2015. Corporinoquia, casanare, yopal.

El Espectador. $(25$ de diciembre de 2015). Pobladores de choachí están preocupados por los efectos de la obra 
sobre el agua y los bosques nativos. La agencia nacional de infraestructura argumenta que el manejo ambiental será adecuado. (s. P. Montoya, ed.) Los desacuerdos por la vía perimetral de oriente, pág. 1. Recuperado de http:// www.elespectador.com/noticias/bogota/ los-desacuerdos-via-perimetral-deoriente-articulo-607685

Mavd. (4 de junio de 2007). Resolución 964 de 2007 ministerio de ambiente, vivienda $y$ desarrollo territorial. Recuperado de diario oficial 46649 de junio 04 de 2007: http://www.alcaldiabogota.gov. co/sisjur/normas/norma1.jsp?i=27717

\section{Ministerio de Ambiente Vivienda y Desarrollo}

Territorial mavdt. (2 de junio de 2004). Resolución 0643. Recuperado de diario oficial 45.576: http://www.corpamag. gov.co/archivos/normatividad/ resolucion643_20040602.htm

Miranda, d. \&. (2002). Experiencias y metodología de la investigación participativa. Onu, cepal. Santiago de Chile: publicación de las naciones unidas.

Nullvalue. (24 de agosto de 2004). Inversión supera a los aportes. El tiempo. Recuperado de http://www.eltiempo. com/archivo/documento/mam-1594133

Onu. (2002). Cepal.

Plan de accion programado 2015. (2015). Planeación y ejecución. (municipio de ubaque) recuperado de plan de accion programado 2015: http:// ubaque-cundinamarca.gov.co/apc-aa65643963383734623963/lan-de-accion-2015-programado-ubaque.pdf
Pomca. (2006). Capitulo 10. Corporinoquia.

República, c. G. (s.f.). Informes del estado de los recursos naturales. Cgr, cundinamarca. Bogotá: cgr.

Resolución no. 300.41.13.0191 del 27 de febrero de 2013.por medio de la cual se establecenlasdeterminantes ambientales, los requisitos y procedimientos para la formulación, revisión y ajuste de los planes de ordenamiento territorial (pot) y de los plane, no. 300.41.13.0191 (corporinoquia 27 de febrero de 2013).

Tiempo, e. (10 de agosto de 2004). Cundinamarca, redacción. Obtenido de http://www.eltiempo.com/archivo/ documento/mam-1591904

Tiempo, e. (15 de abril de 2015). No hay control eficaz a licencias ambientales, advierte contraloría. El tiempo. Obtenido de http://www.eltiempo. com/estilo-de-vida/ciencia/no-hay-suficiente-control-a-licencias-ambientales/15568115.

Tiempo, e. (15 de abril de 2015). No hay control eficaz a licencias ambientales, advierte contraloría. El tiempo. Obtenido de http://www.eltiempo. com/estilo-de-vida/ciencia/no-hay-suficiente-control-a-licencias-ambientales/15568115.

Ubaque, alcaldía de. (2015). Informe de gestión 2014. Alcaldía de ubaque, cundinamarca. Ubaque: alcaldía de ubaque.

Umata, d. D. (13 de mayo de 2015). (investigadores, entrevistador) 